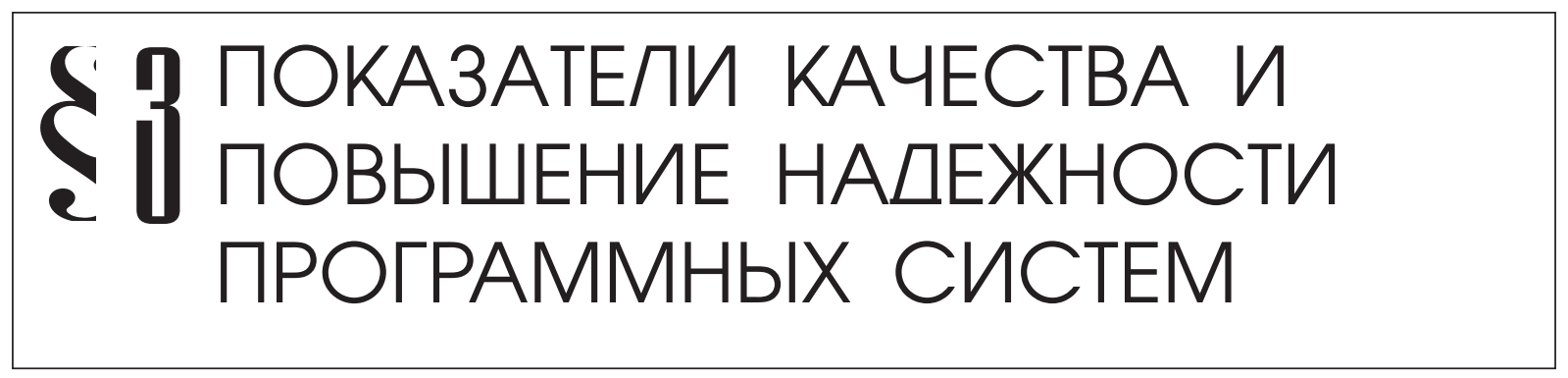

Бойков С.А.

\title{
ЭКСПЕРТНАЯ ОЦЕНКА ФУНКЦИОНАЛЬНОЙ ПОЛНОТЫ АВТОМАТИЗИРОВАННЫХ ИНФОРМАЦИОННЫХ СИСТЕМ ДЛЯ ГОСУДАРСТВЕННЫХ УЧРЕЖДЕНИЙ
}

\begin{abstract}
Аннотация: Предметом исследования в статье являются методы определения функциональной полноты автоматизированных информационных систем, внедряемых в государственных учреждениях социальной сферы. Определяется перечень автоматизируемых функций, оказывающих непосредственное влияние на эффективность деятельности учреждении. В статье рассматривается использование методики, основанной на дельфийском подходе к экспертной оценке. Происходит рассмотрение преимуществ использования данной методики, а также коэффициента парной корреляции Спирмена при определении степени сходимости экспертных оченок. Применение методики демонстрируется на выборке из 8 различных программных продуктов для автоматизации деятельности по оказанию услуг государственными учреждениями социальной сферы. Проведение предварительного экспертного анализа эффективности функций типовой системы согласно методике, основанной на дельфийском подходе к экспертной оценке В данной статье впервые предлагается использование для оценки качества и функциональной полноты автоматизированных информационных систем, внедряемых в государственные учреждения сочиальной сферы, методики, основанной на дельфийском подходе с применением коэффициента парной коррелячии Спирмена при определении степени сходимости экспертных оценок. Проведенный в данной статье анализ показывает, что применение данной методики позволяет существенно повысить объективность оценки, за счет использования обратной связи, анализа результатов предыдущих туров, а также их учета при оценке значимости мнений экспертов.

Ключевые слова: функциональная полнота, экспертная оченка, информационные системы, метод Дельфи, коэффициент Спирмена, методы экспертной оченки, сходимость экспертных оченок, коэффичиент парной корреляции, коэффичиент конкордации, коэффичиент Кендалла
\end{abstract}


При выборе автоматизированной информационной системы, прежде всего, происходит сравнение нескольких аналогичных программных продуктов между собой по цене и по количеству функций. Однако зачастую максимальное количество функций еще не является показателем функциональной полноты информационных систем. Важно определить максимальное соответствие автоматизируемых функций организации функциональным возможностям внедряемой информационной системы.

С целью улучшения оценки потребительского качества АИС в статье предлагается проведение предварительного анализа программного продукта на наличие функций, существенно влияющих на эффективность работы государственного учреждения. Для этого необходимо сопоставить функции рассматриваемой ИС по отношению к рейтингу данных функций в типовой системе. Типовой системой будем считать систему, в которой реализованы все функции, оказывающие влияние на эффективность деятельности учреждения. Пусть в типовой системе каждая функция имеет свой вес. Вес показывает степень важности функции в повышении показателя эффективности работы организации. Все функции системы располагаются в порядке уменьшения их значимости и вес каждой них равен $X_{i}(i=1,2,3, \ldots, k)$, где $k$-общее число функций типовой системы.

В соответствии с данными весами, проведем оценку каждой функции рассматриваемой ИС. Сумма весов функций даст итоговую оценку системы по функциональной полноте и способности повысить эффективность работы государственного учреждения.

На этапе проектирования АИС, когда наиболее актуальной становится проблема выбора автоматизируемых функций управления, нет фактических данных, позволяющих оценить показатели эффективности, обусловленные информационными потерями от функциональной неполноты рассматриваемой системы. В данном случае целесообразным является проведение предварительного экспертного анализа эффективности функций типовой системы согласно методике, основанной на дельфийском подходе к экспертной оценке. Преимуществом данного подхода является полный отказ от коллективных обсуждений системы в пользу получения независимых индивидуальных оценок, с целью уменьшения таких психологических факторов, как присоединение кмнению большинства, к мнению наиболее авторитетного эксперта и т.д.

Можно выделить следующие этапы процедуры экспертной оценки по методу Дельф:

1. Формирование рабочей группы. Необходимость в формировании рабочей группы отпадает, если результаты экспертного опроса будут обрабатываться программой.

2. Формирование экспертной группы. В состав данной группы могут входить руководители государственных учреждений, а также руководители управлений, ответственных за деятельность данных организаций.

3. Определение автоматизируемых функций управления.

4. Проведение экспертного опроса.

5. Подведение итогов экспертного опроса.

Методика экспертной оценки автоматизируемых функций состоит в следующем. Пусть $m$ - количество экспертов; $n$ - количество автоматизируемых функций, входящих в состав типовой системы, предоставленных эксперту вместе с информацией о затратах 
на их реализацию; $c_{i j}$ - ранг, поставленный $i$-м экспертом $j$-й функции.

В каждом туре эксперт определяет ранг каждой функции, принимая во внимание такие факторы как степень важности функции для увеличения эффективности работы учреждения и затраты на ее реализацию. Ранги, определенные экспертами, представляются в виде матрицы упорядочения. В конце тура оценивается согласованность экспертных мнений. Для оценки степени сходимости используется коэффициент парной корреляции Спирмена, определяющийся по формуле:

$$
r=1-\frac{6 \sum_{j=1}^{n} d_{j}^{2}}{n^{3}-n}
$$

где $d$ - разница между рангами, данными $j$-й функции двумя экспертами.

В результате анализа деятельности государственных учреждений в социальной сфере были выделены следующие функции управления, влияющие на эффективность деятельности учреждения (Таблица 1).

Таблица 1. Автоматизируемые функции управления в государственных учреждениях социальной сферы

\begin{tabular}{|c|c|c|}
\hline $\mathbf{N}$ & Наименование блока функций & Обозначение \\
\hline 1. & Формирование личных дел & P1 \\
\hline 2. & Формирование назначений & $\mathrm{P} 2$ \\
\hline 3. & Ведение статистики и отчетности & P3 \\
\hline 4. & Формирование документов на социальное обслуживание & P4 \\
\hline 5. & Ведение паспорта учреждения & P5 \\
\hline 6. & Формирование начислений и выплат & P6 \\
\hline 7. & Формирование лицевых счетов & P7 \\
\hline 8. & Формирование заявлений & P8 \\
\hline 9. & Регистрация оказания государственных услуг & P9 \\
\hline 10. & Информационный обмен & P10 \\
\hline
\end{tabular}

Результаты опроса показали удовлетворительную сходимость мнений экспертов относительно важности данных функций после проведения 3 туров. Сводные данные опроса экспертов после 3 тура представлены в таблице 2.

Таблица 2. Сводные данные опроса экспертов после 3 тура по автоматизируемым функциям

\begin{tabular}{|c|c|c|c|c|c|c|c|c|c|c|}
\hline Эксперт & E1 & E2 & E3 & E4 & E5 & E6 & E7 & 焉 & 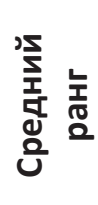 & 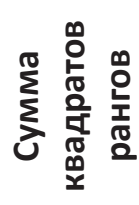 \\
\hline P1 & 1 & 1 & 1 & 1 & 1 & 1 & 1 & 7 & 1 & 49 \\
\hline P2 & 4 & 4 & 5 & 4 & 4 & 4 & 4 & 29 & 4,14 & 841 \\
\hline
\end{tabular}




\begin{tabular}{|c|c|c|c|c|c|c|c|c|c|c|}
\hline Эксперт & E1 & E2 & E3 & E4 & E5 & E6 & E7 & 旁 & 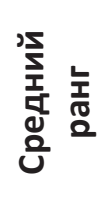 & 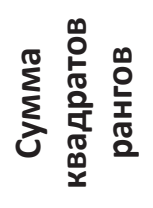 \\
\hline P3 & 8 & 9 & 8 & 7 & 8 & 8 & 8 & 56 & 8 & 3136 \\
\hline P4 & 2 & 2 & 2 & 2 & 3 & 2 & 2 & 15 & 2,14 & 225 \\
\hline P5 & 10 & 10 & 10 & 9 & 10 & 10 & 10 & 69 & 9,86 & 4761 \\
\hline P6 & 6 & 5 & 4 & 6 & 5 & 5 & 5 & 36 & 5,14 & 1296 \\
\hline P7 & 5 & 6 & 6 & 5 & 6 & 6 & 6 & 40 & 5,71 & 1600 \\
\hline P8 & 3 & 3 & 3 & 3 & 2 & 3 & 3 & 20 & 2,86 & 400 \\
\hline P9 & 7 & 7 & 7 & 8 & 7 & 7 & 7 & 50 & 7,14 & 2500 \\
\hline P10 & 9 & 8 & 9 & 10 & 9 & 9 & 9 & 63 & 9 & 3969 \\
\hline Общая оценка & & & & & & & & 385 & & 18777 \\
\hline
\end{tabular}

В таблице 3 представлена матрица рассогласования оценок экспертов после 3 тура.

Таблица 3. Матрица рассогласования оценок экспертов после 3 тура

\begin{tabular}{|l|l|l|l|l|l|l|}
\hline 0 & 4 & 4 & 4 & 4 & 2 & 2 \\
\hline 4 & 0 & 4 & 8 & 4 & 2 & 2 \\
\hline 4 & 4 & 0 & 8 & 4 & 2 & 2 \\
\hline 4 & 8 & 8 & 0 & 8 & 6 & 6 \\
\hline 4 & 4 & 4 & 8 & 0 & 2 & 2 \\
\hline 2 & 2 & 2 & 6 & 2 & 0 & 0 \\
\hline 2 & 2 & 2 & 6 & 2 & 0 & 0 \\
\hline
\end{tabular}

Пороговое значение $e_{d}=160 / 49=3,27$.

Для проверки согласованности оценок экспертов рассчитываем коэффициент конкордации рангов Кендалла по формуле:

$$
W=\frac{12 D}{k^{2} n\left(n^{2}-1\right)}
$$

где $k$ - число экспертов, $n$ - число сравниваемых характеристик, $D$ - сумма квадратов рангов, рассчитываемая по формуле:

$$
D=\sum_{i=1}^{n} r_{i j}^{2}-\frac{\left[\sum_{i=1}^{n} r_{i j}\right]^{2}}{n}
$$

где $r_{i j}$ - ранг, поставленный $i$-той функции $j$-тым экспертом. 
Сумма квадратов рангов для автоматизируемых функций ИС $D=18777-3852 / 10=18777-14822,5=3954,5$.

Коэффициент конкордации рангов Кендалла $W=47454 / 48510=0,978$. Полученное значение свидетельствует о высоком уровне согласованности экспертов (при уровне доверительной вероятности $=0,05)$.

Для выделения согласованных групп экспертов и анализа рассогласования построим матрицу согласования $P_{L T}^{0}$ в канонической форме. Данная матрица представлена в таблице 4:

Таблица 4. Матрица $P_{L T}^{0}$

\begin{tabular}{|l|l|l|l|l|l|l|}
\hline 1 & 0 & 0 & 0 & 0 & 1 & 1 \\
\hline 0 & 1 & 0 & 0 & 0 & 1 & 1 \\
\hline 0 & 0 & 1 & 0 & 0 & 1 & 1 \\
\hline 0 & 0 & 0 & 1 & 0 & 0 & 0 \\
\hline 0 & 0 & 0 & 0 & 1 & 1 & 1 \\
\hline 1 & 1 & 1 & 0 & 1 & 1 & 1 \\
\hline 1 & 1 & 1 & 0 & 1 & 1 & 1 \\
\hline
\end{tabular}

Граф согласования оценок экспертов, построенный на основе матрицы $P_{L T}^{0}$ представлен на рис. 1.

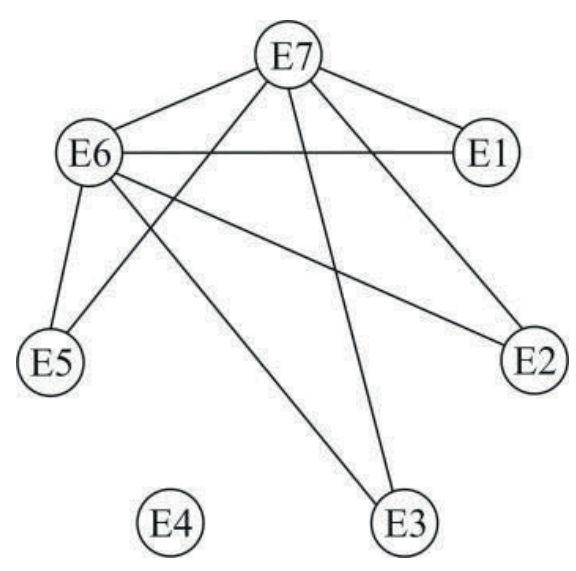

Рис. 1. Граф согласования оценок экспертов

На основе данных матрицы и графа согласования, можно сделать вывод, что в целом наблюдаются схождения в оценках экспертов, относительно важности автоматизируемых функций управления.

Далее определяем весовые характеристики автоматизируемых функций ИС для государственных учреждений социальной сферы (Таблица 5). 
Таблица 5. Весовые характеристики автоматизируемых функций ИС для государственныхучреждений социальной сферы

\begin{tabular}{|c|c|c|c|}
\hline Группа функций & $M_{i}$ & $L_{i}=M_{\text {min }} / M_{j}$ & $W_{i}$ \\
\hline P1 & 1 & 1 & 0,344 \\
\hline P2 & 4,14 & 0,242 & 0,083 \\
\hline P3 & 8 & 0,125 & 0,043 \\
\hline P4 & 2,14 & 0,467 & 0,161 \\
\hline P5 & 9,86 & 0,101 & 0,035 \\
\hline P6 & 5,14 & 0,195 & 0,067 \\
\hline P7 & 5,71 & 0,175 & 0,06 \\
\hline P8 & 2,86 & 0,35 & 0,12 \\
\hline P9 & 7,14 & 0,14 & 0,048 \\
\hline P10 & 9 & 0,111 & 0,038 \\
\hline$\sum$ & 55 & 2,906 & 1 \\
\hline
\end{tabular}

Так как данные функциональные блоки могут включать несколько функций, то функциональная полнота рассматриваемой ИС будет определяться по формуле:

$$
W_{S}=\sum_{i=1}^{n} \frac{W_{i}}{k_{i}} \cdot c_{i},
$$

где $k_{i}$ - количество функций, входящих в функциональный блок, $c_{i}$-количество функций из функционального блока, реализованных в рассматриваемой ИС.

На основе полученных результатов согласования оценок экспертами и весовых характеристик автоматизируемых функций, можно получить значения функциональной полноты систем, а также проводить сравнение рассматриваемых систем по данному критерию, как между собой, так и по отношению к типовой системе. Пример результатов применения данной методики по отношению к АИС для государственных учреждений в социальной сфере приведен в таблице 6.

Таблица 6. Весовые характеристики ИС для государственных учреждений социальной сферы

\begin{tabular}{|c|c|c|c|c|c|c|c|c|}
\hline $\begin{array}{l}\text { Блок } \\
\text { Функций }\end{array}$ & 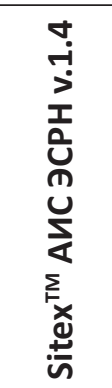 & 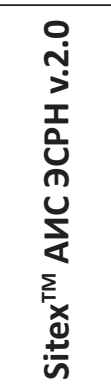 & 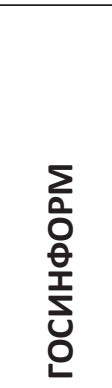 & 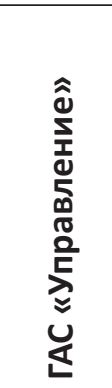 & 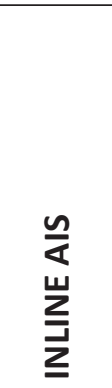 & 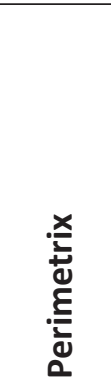 & 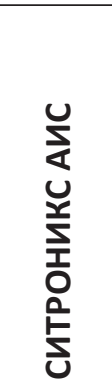 & 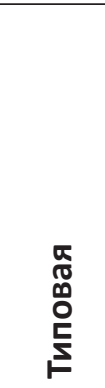 \\
\hline P1 & 0,275 & 0,275 & 0,206 & 0,241 & 0,31 & 0,206 & 0,172 & 0,344 \\
\hline P2 & 0,083 & 0,083 & 0,083 & 0,083 & 0,083 & 0,083 & 0,083 & 0,083 \\
\hline
\end{tabular}




\begin{tabular}{|c|c|c|c|c|c|c|c|c|}
\hline $\begin{array}{l}\text { Блок } \\
\text { функций }\end{array}$ & 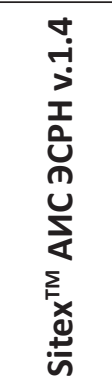 & 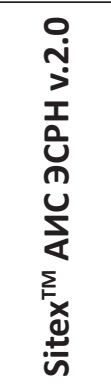 & $\begin{array}{l}\sum \\
0 \\
0 \\
\hat{\theta} \\
\text { 언 } \\
\text { 은 }\end{array}$ & 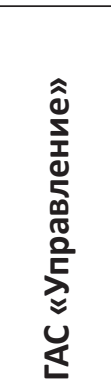 & 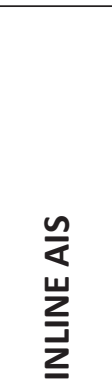 & 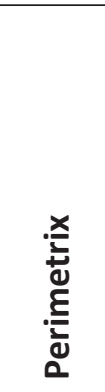 & 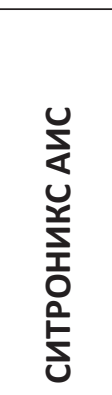 & 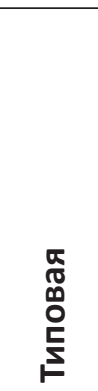 \\
\hline P3 & 0,029 & 0,029 & 0,043 & 0,029 & 0,029 & 0,029 & 0,029 & 0,043 \\
\hline P4 & 0,161 & 0,161 & 0,161 & 0,161 & 0,161 & 0,161 & 0,161 & 0,161 \\
\hline P5 & 0,014 & 0,028 & 0,007 & 0,007 & 0,007 & 0,007 & 0,007 & 0,035 \\
\hline P6 & 0,067 & 0,067 & 0,045 & 0,045 & 0,045 & 0,045 & 0,045 & 0,067 \\
\hline P7 & 0,06 & 0,06 & 0 & 0 & 0 & 0 & 0 & 0,06 \\
\hline P8 & 0,12 & 0,12 & 0,12 & 0,12 & 0,12 & 0,12 & 0,12 & 0,12 \\
\hline P9 & 0,032 & 0,048 & 0,032 & 0,032 & 0,032 & 0,032 & 0,048 & 0,048 \\
\hline P10 & 0,038 & 0,038 & 0,019 & 0,019 & 0,019 & 0 & 0,019 & 0,038 \\
\hline $\begin{array}{c}\text { Итоговый вес } \\
\text { ИС: }\end{array}$ & 0,879 & 0,909 & 0,716 & 0,736 & 0,805 & 0,683 & 0,683 & 1 \\
\hline
\end{tabular}

Сравнительная диаграмма ИС для государственных учреждений социальной сферы по критерию функциональной полноты представлена на рисунке 2.

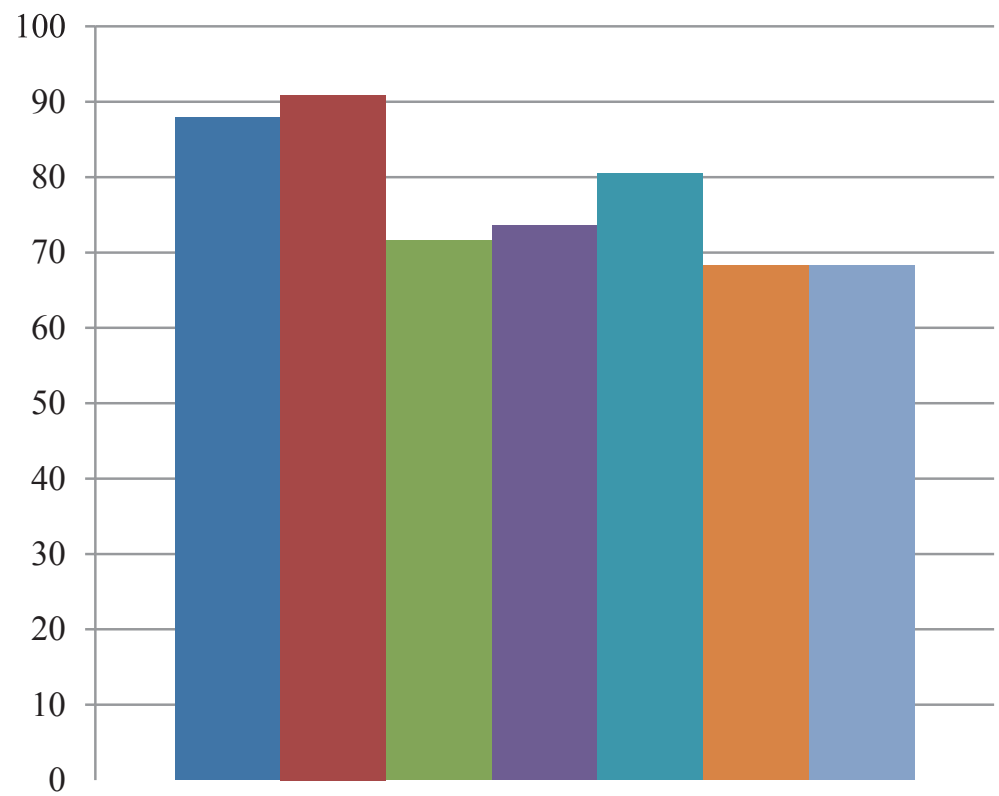

- SitexТМ АИС ЭСРН v.1.4

$\square$ SitexTM АИС ЭСРН v.2.0

- ГОСИНФОРМ

- ГАС «Управление»

- INLINE AIS

- Perimetrix

- СИТРОНИКС АИС

Рис. 2. Функциональная полнота АИС в процентном отношении

По полученным результатам можно сделать Вывод, что наибольшей функциональной полнотой обладают системы АИС ЭСРН v.1.4 и АИС ЭСРН v.2.0. 
Проведенный в данной статье анализ показывает, что применение комбинированного метода на основе метода Дельфи и метода парных сравнений при экспертном оценивании функциональной полноты автоматизированных информационных систем позволяет существенно повысить объективность оценки, за счет использования обратной связи, анализа результатов предыдущих туров, а также их учета при оценке значимости мнений экспертов.

\section{Библиография :}

1. Литвак Б.Г. Экспертные технологии в управлении: Учеб. Пособие.-М.: Дело, 2004.-400 С.

2. Орлов А.И. Экспертные оценки. Учебное пособие.-М.: 2002.-31 С.

3. Хубаев Г.Н. Получение групповой экспертной оценки значений показателей: пошаговая процедура и программное обеспечение // Программные продукты и системы.-2011.-№2.-С. 2-8.

4. Хубаев Г.Н. Сложные системы: экспертные методы сравнения // Изв. вузов. Северо-Кавказский регион. 0бщественные науки. 1999. № 3. С. 7-24.

\section{References:}

1. Litvak B.G. Ekspertnye tekhnologii v upravlenii: Ucheb. Posobie.-M.: Delo, 2004.-400 S.

2. Orlov A.I. Ekspertnye otsenki. Uchebnoe posobie.-M.: 2002.-31 S.

3. Khubaev G.N. Poluchenie gruppovoi ekspertnoi otsenki znachenii pokazatelei: poshagovaya protsedura i programmnoe obespechenie // Programmnye produkty i sistemy.-2011.-№2.-S. 2-8.

4. Khubaev G.N. Slozhnye sistemy: ekspertnye metody sravneniya // Izv. vuzov. Severo-Kavkazskii region. Obshchestvennye nauki. 1999. № 3. S. 7-24 